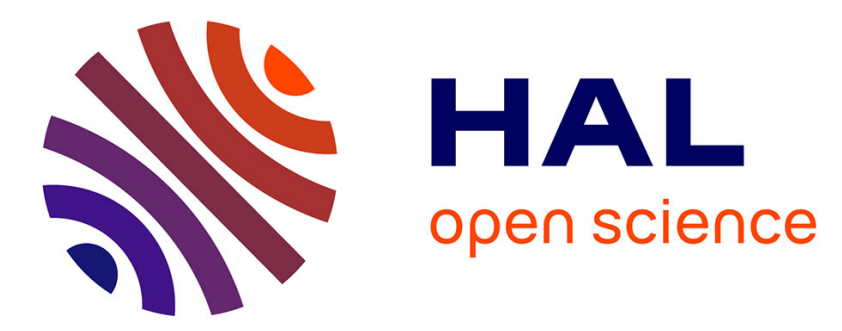

\title{
Magnetic miniature swimmers with multiple rigid flagella
}

Johan Quispe, Stéphane Régnier

\section{To cite this version:}

Johan Quispe, Stéphane Régnier. Magnetic miniature swimmers with multiple rigid flagella. 2020 IEEE International Conference on Robotics and Automation (ICRA), May 2020, Paris, France. pp.9237-9243, 10.1109/ICRA40945.2020.9196531 . hal-03373418

\section{HAL Id: hal-03373418 https://hal.science/hal-03373418}

Submitted on 11 Oct 2021

HAL is a multi-disciplinary open access archive for the deposit and dissemination of scientific research documents, whether they are published or not. The documents may come from teaching and research institutions in France or abroad, or from public or private research centers.
L'archive ouverte pluridisciplinaire HAL, est destinée au dépôt et à la diffusion de documents scientifiques de niveau recherche, publiés ou non, émanant des établissements d'enseignement et de recherche français ou étrangers, des laboratoires publics ou privés. 


\title{
Magnetic miniature swimmers with multiple rigid flagella
}

\author{
Johan Quispe and Stéphane Régnier
}

\begin{abstract}
In this paper, we introduce a novel miniature swimmer with multiple rigid tails. The tails' geometry is based on spherical helices that benefit the swimmers for transporting objects with their flagellar bundle. When the swimmer is rotated, their tails provide a considerable propulsive force to generate a net displacement. Thus, achieving propulsion speeds up to $6 \mathrm{~mm} / \mathrm{s}$ at $3.5 \mathrm{~Hz}$ (small rotation frequencies) for a $6-\mathrm{mm}$ in size prototypes. We study the efficiency of different bundle distribution for a 2-flagella swimmer by varying the phase angle between tails. Moreover, it is demonstrated that these swimmers experience a great sensibility when changing their tail height. Besides, the swimmers demonstrate to be effective for cargo carrying tasks since they can displace objects up to 3.5 times their weight. Finally, the confinement effect is studied with multi-tailed swimmer robots considering 2 containers with 20 and 50-mm in width. Results showed speeds' increments up to $59 \%$ when swimmers are actuated in the smaller container.
\end{abstract}

\section{INTRODUCTION}

Over the years, the microworld has drawn much attention of various research areas because of its fascinating and nonintuitive way of working. At small scales, inertia and gravity forces are less significant. Conversely, surfaces forces play an important role for microorganism propulsion. These species must perform nonreciprocal patterns in order to propel [12]. Multifarious of such species are provided of single or multiple flagella through an evolutionary process where optimization was performed through survival and reducing energy consumption [11]. Moreover, microorganisms developed different propulsion mechanisms, namely, a bundle rotating in a corkscrew fashion or a beating flagellum propelling by planar undulations. The former is inspired by prokaryotic cells such as E. coli bacteria, R. sphaeroides, Spiroplasma, and so on [9]. During last decades, single helical flagellum swimming have been well reviewed in the literature. Great efforts have been made to find an optimal shape for the single flagellum. The influence on the swimming efficiency of parameters such as the pitch [17], pitch angle [18], [16], number of turns [17], total length [4], magnetization [7], flagellum thickness [17], and so on have been widely studied through experiments and simulations [8], [2], [11], [14]. This trend of studying the single-flagellum propulsion born because real microorganisms present multiple flagella that frequently generate a single rotating bundle to self-propel. Nonetheless, organisms such as magnetotactic bacteria include the strain MO-1 that generates two bundles which rotate independently from well-defined locations [10]. Each

\footnotetext{
*This work was supported by the French National Research Agency through ANR MultiFlag (grant number ANR-16-CE33-0019).

Johan Quispe and Stéphane Régnier are with the Institut des Systèmes Intelligents et Robotique (ISIR), Sorbonne Université, 75005 Paris, France. quispedisir.upmc.fr
}

bundle is composed of 7 flagellar filaments and numerous fibrils enveloped in a sheath [13]. The main advantage is that this structure can propel the bacterium at speeds of up to $300 \mu \mathrm{m} / \mathrm{s}$, an order of magnitude faster than many other flagellated bacteria. In [14] is demonstrated that this structure with two bundles achieves a better propulsion speed when bundles are far apart. In addition, this 2-bundle prototype is proven to escape with ease from surfaces, conversely to their single-bundle counterparts. Other microorganisms such as Paramecium have independent beating cilia around their plasma membranes that allow them to self-propel [9]. Previous studies have demonstrated propulsion enhancement by increasing the number of flexible flagella [19], [15]. In those works, straight-flexible flagella were manufactured and placed on a magnetized head with an offset distance and an anchoring angle with respect to the robot's axis of rotation. When the robot's body rotates, their tails experienced bending angles achieving a chiral structure, and thus provoking a net axial force to propel in the viscous medium. The authors in [5] studied bio-hybrid microswimmers propelled by multiple bacterial cells. Such swimmers consist of Serratia marcescens bacteria attached to a $6 \mu \mathrm{m}$-diameter superparamgnetic bead. Authors demonstrated that using remote magnetic control reduces the stochasticity of motion, enabling steering control. Besides, a direct proportion between the number of attached bacteria and the reached speed is proven as well. Notwithstanding these results, the bundle shapes was studied in [6] considering rigid millimetric prototypes using CFD simulations and PIV techniques. The authors observed that the most efficient configuration is for a single flagellum. Furthermore, they showed that 2-flagella configurations with 90 and 180 degrees in phase (angular tail separation) are ineffective, conversely to previous results considering flexible tails. In [2], using bead-shell formulation the authors found different optimal structures considering the same volume and varying the flagellum helical radius and pitch angle. Some of these optimal structures are not found in nature, bringing new criteria for fabricating artificial microrobots.

In this article, miniature robot designs with multiple flagella are introduced. Such structures consider some of the previous discussed points, namely, a multi-flagellated structure, a chiral shape inspired by spherical helices, and a large helical radius, in such a manner to create a shape not seen before in nature but with effective propulsion. Although their geometries are quite different from real micro-species, their propelling mechanism remains the same. The experiments with these millirobots were performed in a low Reynolds regime using pure glycerol. However, even if gravity force 


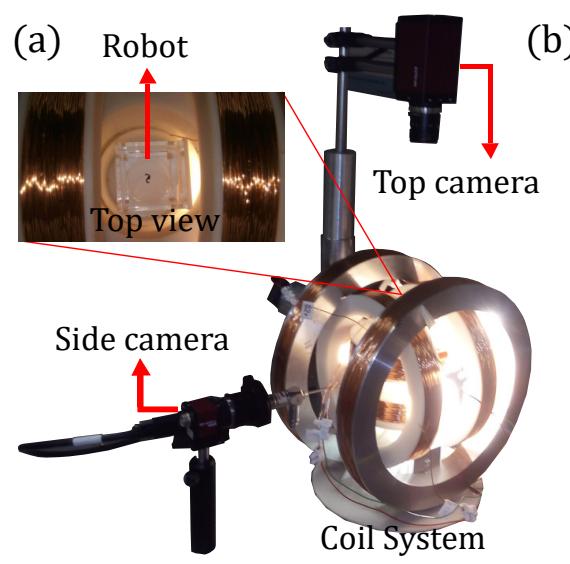

(b)

Fig. 1. Experimental set-up and swimmer prototype. (a) The system is mainly composed of a side camera with an endoscope, a top camera, and a 3D Helmholtz coil system for the magnetic actuation. (b) The swimmer prototype is composed of multiple 3D printed tails with a disc magnet placed inside the cylindrical head.

at this regime is smaller than viscous forces, the gravity term will act as a perturbation term for the swimmer dynamics. We study the bundle distribution of a 2-flagella swimmer finding an optimum distribution when tails are separated a phase angle of $180^{\circ}$. Moreover, the in-phase distribution (phase angle $0^{0}$ ) is ineffective since the swimmer does not achieve to propel, confronting the results in [6] with the typical helical geometries. Furthermore, the proposed 2-flagella swimmer with 3-mm in tail height demonstrates to be susceptible to tail height variations. Results demonstrate that just an small increment of 1-mm of the height can duplicate the speed while the decrease of 1-mm can cause a null propulsion speed. In addition, this swimmer could be helpful for cargo carrying applications. Its great helical radius allow it to carry objects with even 3.5 times its weight. Finally, the last contribution relies on the analysis of the confinement effect for different symmetric bundle distributions of 2, 3 and 4 tails in 2 containers with 20 and $50-\mathrm{mm}$ in width. The swimmers in all cases achieve increments on propulsion speed, reaching an increment up to $59 \%$ for a 3 -flagella robot. In this study as well, it is demonstrated that the 2-flagella distribution is the most effective for this class of robots.

The manuscript is organised as follows: in section II, the conception and actuation of the robot are presented. Moreover, the theoretical background for modeling the proposed robot is treated with finite element method (FEM) formulation through a commercial software, COMSOL Multiphysics. In Section III, we present simulation and experimental results accompanied by a brief discussion. Results are related to geometric optimization, maximum cargo, and confinement effect experimented by the proposed robot. Finally, in section IV, we synthesize discussion preceding of conclusions, and perspectives for future work.

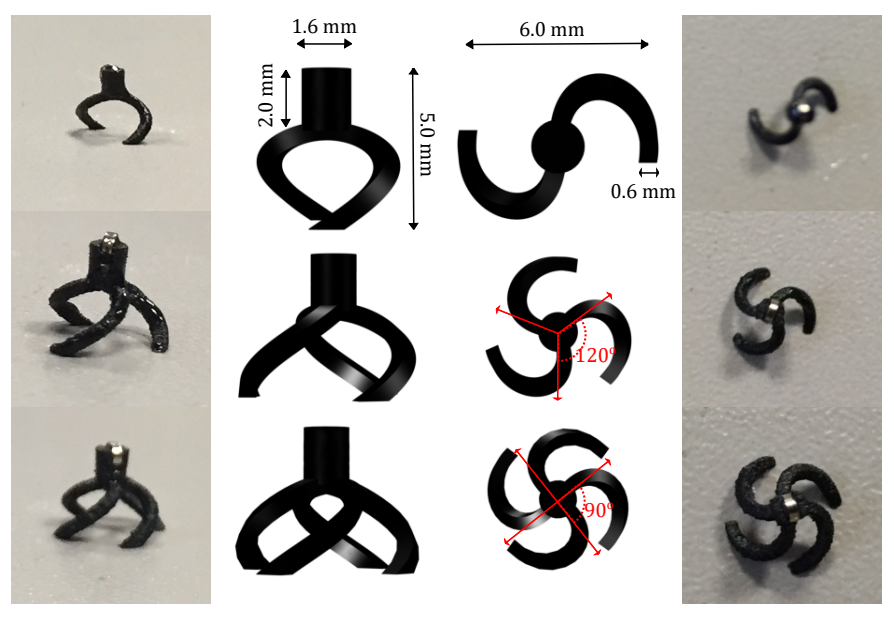

Fig. 2. Robot designs with multiples tails 2,3 and 4 . The magnet is found inside the cylindrical head with $1.6 \mathrm{~mm}$ in diameter and $2 \mathrm{~mm}$ in height. The real printed prototypes are flanked respectively the CAD's. For the case study with finite elements we use the 2-tailed robot, the rest is used for the confinement effect experiments.

\section{CONCEPTION, MODELING AND SIMULATION}

\section{A. Conception and actuation}

The millimetric prototypes are composed of spherical helical tails conceived by 3D printing with Visijet M3 black as a UV curable plastic material. The magnetization is given through a disc-shaped neodymium magnet $(\phi=1.0 \mathrm{~mm}$ in diameter, and $\delta d=0.5 \mathrm{~mm}$ in thickness) attached to a cylindrical head (cf. figure 1.b). The tail geometry follows the next parameterization:

$$
\begin{gathered}
x=\sqrt{R^{2}-t^{2}} \cos (t / s) \\
y=\sqrt{R^{2}-t^{2}} \sin (t / s) \\
z=t
\end{gathered}
$$

This parameterization stands for a helical flagellum drawn on a spherical surface with radius $R$. Parameters $t$ and $s$ determine the number of turns and helix curvature. In most of experiments we keep $R=3$, except for the experiments concerning the tail height. Variable $t$ is strong correlated with $R$, and in order to not obtain a complex number in the parameterization, $t$ might range from 0 to $R$. Finally, by convenience we kept the parameter $s$ equal to $R$ in all experiments, thus maintaining helix shape for the tail height experiments. The experiments were carried out in pure glycerol with the finality of reproducing a low Reynolds environment. The robots are guided harnessing magnetic fields generated by a 3D nested Helmholtz coil system (cf. figure 1.a). The maximum magnetic field achieved by the system is $12 \mathrm{mT}$. However, the robots were just actuated by $8 \mathrm{mT}$ rotating magnetic field to avoid high temperatures in the workspace.

\section{B. Modeling and simulation - finite element formulation}

The finite element method is an alternative discretization method for solving partial differential equations (PDEs). 


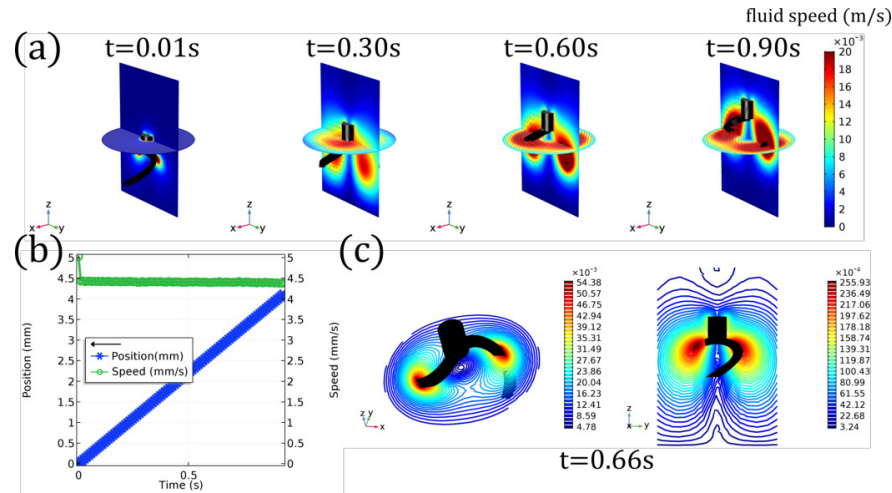

Fig. 3. Kinematics of the 2-flagella robot. (a) Depicts the robot displacement and the fluid speed around for 4 frames.(b) shows a graph representing the position and speed reached during 0.9 seconds. (c) Draw the isocurves of speed for the top and side views.

This method approximates the solutions of these PDEs by subdividing a large system into smaller, simpler parts that are called finite elements.

A priori, the problem that we face involves fluid-structure interaction (FSI) formulation since the swimming robot rotates around its axis generating a net displacements in a viscous fluid. Nonetheless, this interaction is just one-way coupled. In other words, since the robot has a rigid structure, fluid will not be able to deform it. Hence, only the fluid deformation caused by the rotation of the rigid structure is considered. This simplification tremendously reduced the computation time. The one-way coupled FSI problem groups Navier-Stokes $(\mathrm{N}-\mathrm{S})$ equations with the mesh treatment. N-S equations ( $\Omega_{f}$ fluid domain) solves the fluid dynamics, the rotating structure dynamic ( $\Omega_{s}$ structure domain) is solved by mesh equations that deal with mesh translation and deformation. The boundary condition ( $\partial \Omega_{f s}$ fluid-structure boundary) on the robot-fluid interface relates mesh translation and fluid dynamics through all time evolution ( $\tau$ time interval). $\mathrm{N}$ $\mathrm{S}$ equations are composed of the momentum conservation and the mass conservation equations. They are expressed as follows:

$$
\begin{gathered}
\frac{\partial \mathbf{u}}{\partial t}+\rho(\mathbf{u} . \nabla) \mathbf{u}=\nabla . \Gamma+\mathbf{F} \text { in } \Omega_{f} \times \tau \\
\frac{\partial \rho}{\partial t}+\nabla(\rho . \mathbf{u})=0 \text { in } \Omega_{f} \times \tau \\
\Gamma=-p \mathbf{I}+\mu\left(\nabla \mathbf{u}+(\nabla \mathbf{u})^{T}\right)
\end{gathered}
$$

With $\mathbf{u}$ as the fluid velocity, $p$ pressure, $\rho$ density, $\mu$ dynamic viscosity, $\Gamma$ depicting the stress on an infinitesimal fluid volume ( $\nabla . \Gamma$ surface forces) and $\mathbf{F}$ the volume forces.

Concerning the rigid robot structure, a rotation movement is along the robot helix axis. That can be translated in a prescribed mesh motion $(d \mathbf{X})$ since there is no structure deformation.

$$
d \mathbf{X}=d \mathbf{X}(\mathbf{r}, \omega, t) \text { in } \Omega_{m} \times \tau
$$

Where $\mathbf{r}$ is the helix axis, $\omega$ is the angular frequency and $t$ is time and $\Omega_{m} \subset \Omega_{s}$. The boundary condition in $\partial \Omega_{f s}$ (a)
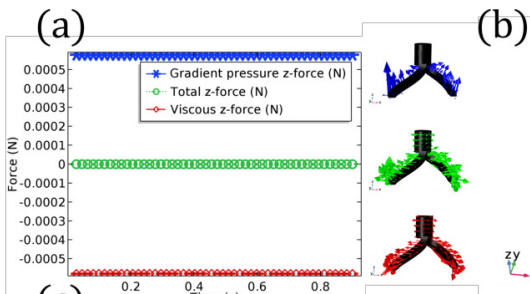

(b)

(c)

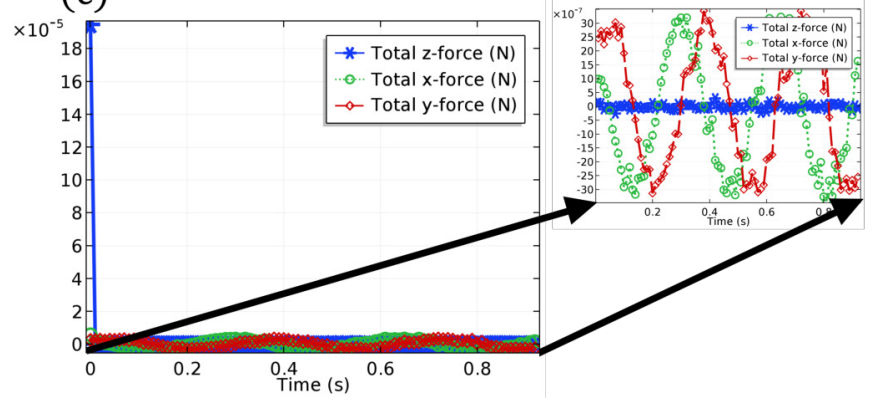

Fig. 4. Dynamics of the 2-flagella robot. (a) separates the total force in $\mathrm{z}$ (green) in its pressure (blue), viscous (red) components. (b) Depicts how the viscous force (red arrows on the robot's surface) is opposite to fluid velocity. (c) shows the total force decomposed in its 3 components in $x$, $\mathrm{y}$ and $\mathrm{z}$. While the total force in $\mathrm{z}$-component converges rapidly to zero, the $\mathrm{y}$ and $\mathrm{x}$-components shows an harmonic behaviour because of the tail disposition and symmetry.

that stands for the coupling between N-S equations and the structure movement is given by:

$$
\begin{gathered}
\mathbf{u}=\mathbf{u}_{s} \text { in } \partial \Omega_{f s} \times \tau \\
\mathbf{u}_{s}=\frac{\partial \mathbf{X}}{\partial t} \text { in } \partial \Omega_{f s} \times \tau
\end{gathered}
$$

Where $\mathbf{u}_{s}$ is the solid structure velocity in one point situated on its surface. Another boundary condition in the limits of the container $\left(\partial \Omega_{f}\right)$ is:

$$
\mathbf{u}=\mathbf{0} \text { in } \partial \Omega_{f} \times \tau
$$

Null fluid speed in the fluid-container boundary. Finally, force components are computed by integrating the total stress $(\Gamma)$ over all the robot's surface.

$$
F_{i}=\int_{\partial \Omega_{s}} \Gamma \mathbf{n}_{f} \cdot \mathbf{e}_{i} d s \text { in } \partial \Omega_{f s} \times \tau
$$

Being $\mathrm{i}=\mathrm{x}, \mathrm{y}$, and $\mathrm{z}$ for all different components, $\mathbf{n}_{f}$ the normal vector to the surface of each finite element on the robot surface, $d s$ the surface element, and finally, $\mathbf{e}_{i}$ representing the unit vectors for $\mathrm{x}, \mathrm{y}$, and $\mathrm{z}$ of the global frame. For the robot dynamics we compute the net displacement by using the Newton's second law.

$$
\begin{gathered}
m \frac{d v_{z}}{d t}=F_{z}+g V\left(\rho_{\text {fluid }}-\rho_{\text {robot }}\right) \\
\frac{d x_{z}}{d t}=v_{z}
\end{gathered}
$$

Where $d x_{z}$ is the displacement in the forward direction, $v_{z}$ is the robot speed. The term $g V\left(\rho_{\text {fluid }}-\rho_{\text {robot }}\right)$ (perturbation term) is the buoyancy force, where $g$ is the gravity and $V$ is the robot volume. Forces in $x$ and $y$ are not considered 
(a)

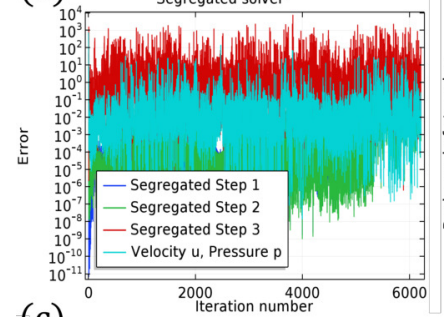

(c)

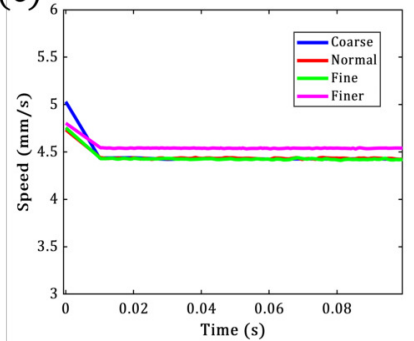

(b)

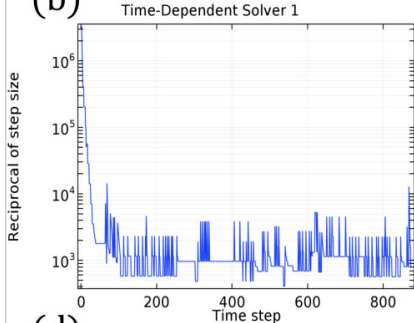

(d)

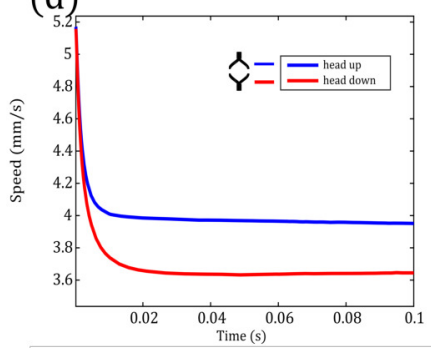

Fig. 5. Convergence study. (a) Segregated solver error. (b) Time-dependent solver, reciprocal time-step. (c) Mesh effect on speed accuracy. (d) effect on speed of having the robot with head up or head down.

because we are only interested in the propulsion direction to obtain the robot's forward speed as well as reduce the computation time. For the first simulation, let's consider as a case study the 2-flagella (cf. figure 2 - first row) robot without considering the perturbation term. In this case we consider a $R$ parameter equal to $3 \mathrm{~mm}$, since the head geometry is $2 \mathrm{~mm}$ in height the robot's total height is 5 $\mathrm{mm}$. Figure 3.a shows image sequences of the evolving kinematics of the robot when rotating at a $3.5 \mathrm{~Hz}$ as well as the change on the fluid velocity field at $\mathrm{t}=0.01,0.30$, 0.60 and $0.90 \mathrm{~s}$. Moreover, the position of the robot center of mass and the speed reached during the time evolution is stand for in figure 3.b. Besides, in fig. 3.c is shown the top and side view of the fluid speed isocurves at $t=0.66 \mathrm{~s}$. On the other hand, the dynamics of this prototype is studied in figure 4. The total z-force (in the propulsion direction, green circles) is decomposed in its viscous (red diamonds) and pressure (blue asterisks) part and depicted in fig. 4.a. The total $\mathrm{z}$-force through the evolving time becomes zero since the viscous forces tend to compensate the gradient pressure forces. Figure 4.b represents the rotating fluid velocity field (magenta arrows) that are opposite to total viscous force (red arrows on robot surface). Finally, the total force is decomposed in its $\mathrm{x}, \mathrm{y}$ and $\mathrm{z}$-components (green, red and blue respectively in fig. 4.c). While the $\mathrm{z}$-component is null through the time evolution, the components in $\mathrm{x}$ and $\mathrm{y}$ have an harmonic behaviour because of the symmetry disposition of the robot's tails. When solving a multiphysics model, there are two approaches that can be taken to solving the (usually nonlinear) system of equations that describe the solution. the fully coupled approach forms a single large system of equations that solve for all the unknowns (the fields) and includes all of the couplings between the unknowns (the multiphysics effects) at once, within a single iteration. On the other hand, the "segregated" approach will not solve

(a) Optimal angular position for a second tail

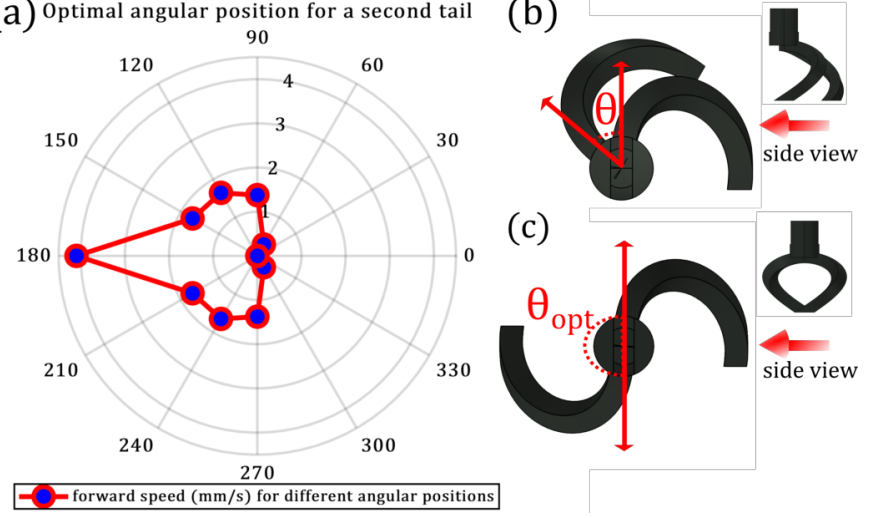

Fig. 6. Looking for the optimal position for a second tail to enhance propulsion. (a) Polar plot depicting the forward speed $(\mathrm{mm} / \mathrm{s})$ in the radial axis vs. angular separation between tails $\theta\left(^{\circ}\right)$. The measurements for this experiment were done 3 times and the error bars were negligible. (b) A sample of a robot with an angular separation of $\theta$. (c) The angle that maximizes propulsion speed is $180^{\circ}$.

for all of the unknowns at one time. Instead, it subdivides the problem up into two or more "segregated steps". These individual segregated steps are smaller than the full system if equations that are formed with the fully coupled approach. The segregated steps are solved sequentially within a single iteration, and thus less memory is required. Figure 5.a depicts the convergence error of the segregated steps per each iteration. In fig. 5.b is shown the reciprocal of step size vs. the time step. Besides, a mesh susceptibility study was performed considering the case study with different mesh sizes: coarse, normal, fine, finer. We analysed the speed evolution during $0.1 \mathrm{~s}$ of the velocity when setting different meshes sizes. These results are shown in fig. 5.c. Moreover, table I gives an extra-information of mesh study concerning the number of elements for each mesh size and the time taken for each simulation in a 28 GB RAM computer with 7 cores. Finally, as an additional study, we considered the case when the robot is propelling in an inverted fashion (with the head up and down). Results shown a decrease in the forward speed that can be caused by the change of the aerodynamic profile when the robot is inverted while going forward.

TABLE I

MESH SENSIBILITY STUDY

\begin{tabular}{cccc}
\hline Mesh & Max. mesh size $(\mathrm{mm})$ & \# of elements & Computation time \\
\hline Coarse & 1.82 & 59310 & $1558 \mathrm{~s}$ \\
Normal & 1.40 & 166946 & $4722 \mathrm{~s}$ \\
Fine & 0.938 & 532977 & $20658 \mathrm{~s}$ \\
Finer & 0.322 & 1438303 & $110035 \mathrm{~s}$ \\
\hline
\end{tabular}

\section{EXPERIMENTAL AND SIMULATION RESULTS}

\section{A. Optimal angular position for a second tail}

Through experimentation, the optimal angular position for a second tail is studied. Considering the proposed tail geometry, we fabricated different 2-flagella robots with different angular separations (cf. fig. 6.b). These robots were actuated under $8 \mathrm{mT}$ rotating magnetic field at $4 \mathrm{~Hz}$. The forward 


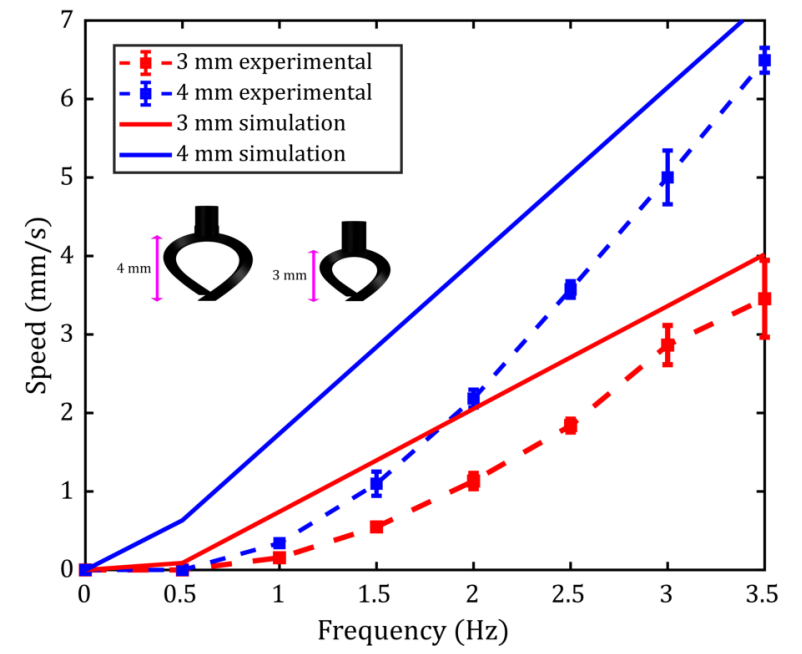

Fig. 7. Analysis of height influence on propulsion speed. Solid lines and dashed lines stand for simulation and experimental results, respectively. Blue and red lines depict results for the 3- $\mathrm{mm}$ and 4-mm in tail length robots, respectively. Simulation and experimental results show an increase of speed when tail height is increased. The error bars in experiments are calculated as the standard deviation of 3 trials for each frequency and robot.

propulsion speed was measured for each configuration 3 times (negligible error) and plotted in polar coordinates in fig. 6.a. The radial axis depicts the forward speed in $(\mathrm{mm} / \mathrm{s})$ while the angular separation is represented by the angles in the graph. Figure 6.b depicts a robot with an angular separation $\theta$ between tails. The tail on the left is kept while the other is rotated forming different angles $\theta$. In the experiments were considered $\theta=0,30,60,90,120,150$, 180 degrees because of the robot symmetry. The in-phase configuration $(\theta=0)$ demonstrates, in this case, to generate insufficient propulsion force to get off the ground. Different from the result obtained in [6]. Finally, in figure 6.c stands for the optimal angular distribution of tails, being 180 degrees the most performant. It means that both tails have to be as far as possible distributed in order to avoid hydrodynamic interactions between each other and thus achieving high performance. A similar result was obtained recently in [14] for a 2-bundled robot with each bundle rotating independently.

\section{B. Height influence}

This study is devoted to analyze the effect on forward speed of the length increment and decrease. The experiments were carried out through finite element simulations and corroborated experimentally. Initially, we started considering 3 robots with tails height of: 2,3 and $4 \mathrm{~mm}$ or more precise with parameter $R=2,3$, and 4. However, the 2-mm robot tails were not able to provide the enough net force to displace the robot. hence, figure 7 only include results for the robots of 3-mm, and 4-mm in tail heights. In both cases, simulations and experiments, the tail length increased the forward speed. The robot in 4-mm tail height experienced an increment of practically $100 \%$ in forward speed with respect to its smaller counterpart just by increasing 1-mm the tail height. On the other hand, if we reduce in $1-\mathrm{mm}$ the

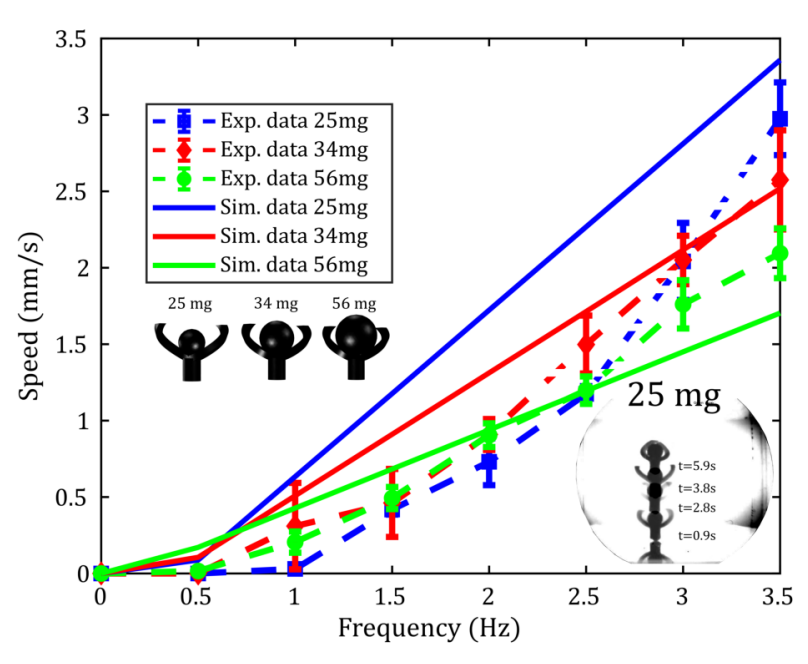

Fig. 8. Cargo influence on propulsion speed. Solid and dashed lines represent simulation and experimental data, respectively. Blue, red and green color represents the cargo weight of 25,34 and $56 \mathrm{mg}$ respectively. Simulation and experimental results have the same trend, the larger cargo amount, the less propulsion speed. The inset on the bottom right shows in the same frame different positions of the robot with the $25 \mathrm{mg}$ cargo. The experimental error bars are calculated as the standard deviation of three trials for each frequency and robot.

tail height of the robot in 3-mm tail height, it will not be able to compensate the robot apparent weight and propulsion becomes non-achievable.

\section{Cargo influence}

As part of the study, the feasibility for cargo transporting was assessed considering spheres with different weights attached inside flagella of a 2-tailed robot with $3-\mathrm{mm}$ in tail height. Simulations and experiments were performed in order to analyze the correlation between the cargo weight and the propulsion speed. The tested weights for this experiment were 25,34 , and $56 \mathrm{mg}$ as can be seen in figure 8 . Results confirm the intuition that the heavier cargo, the less propulsion speed. The interesting part of this is the robot capacity to transport even objects 3.5 times heavier than its weight (2-flagella robot's weight is $16 \mathrm{mg}$ ).

\section{Confinement effect}

Future applications in microrobotics involve medicament delivery in tiny, constrained, and difficult-to-reach environments; where confinement effect will change drastically the robot dynamics. Several studies have stated that confinement effect benefit robot propulsion since speed increases [1], [3]. In spite of this increment, the viscous drag increases as well as the viscous fluidic torque producing a decrease of cutoff frequency in case we set the magnetic torque constant. In other words, in order to avoid a cut-off frequency, we would need to increase the magnetic field value in order to increase as well the magnetic torque and overcome the fluidic torque. The experiment consists of analysing the confinement effect of 3 different multi-flagella robots with 2, 3, and 4 tails arranged symmetrically (cf. figure 2). Each robot is placed in two containers with 20 and $50 \mathrm{~mm}$ in diameter respectively, 
then, actuated in $8 \mathrm{mT}$ rotating magnetic field at frequencies ranging from 0 to $3.5 \mathrm{~Hz}$. The obtained results for containers with $50-\mathrm{mm}$ and $20-\mathrm{mm}$ in diameter are depicted in figure 9.a and 9.b respectively. The results are divided in FEM simulations (solid lines) and experimental (dashed lines). In both cases, comparing frequency by frequency for each robot in each container (before the respective cut-off frequency), speed increases considerably. In the inset of figure 9.b, a bar graph is depicting the increment ratio of the speed of each robot at cut-off frequencies when placed in the $20-\mathrm{mm}$ diameter container. This bar graph shows that the maximum a speed increment is for a 3-tailed robot, achieving a 59\% with respect to the speed when swimming in the 50-mm container. However, as aforementioned, the problem of the cut-off frequencies can be overcome if we increases the magnetic field. Finally, comparing the effectiveness of the multi-tailed robots, we could see that for the 50-mm container the speeds of the 2 and 3-tailed were close. However, when analysing the results for a $20-\mathrm{mm}$ container, the 2-tailed robot resulted the most effective.

\section{Conclusions AND PERSPeCtives}

Through these studies, we analysed the optimal position for a second tail in a miniature robot, the tail height effect on propulsion, the influence of cargo on propulsion, and the boundary effect with rigid multi-flagella prototypes (2, 3 and 4 tails). These studies were performed in most of cases using two approaches: finite element simulations and experimentation. Thus, corroborating the simulation data. We found that the optimal angular position for a second tail in a 2-flagella configuration is $180^{\circ}$, as far as possible from each other. Furthermore, the influence of the tail height was demonstrated to be strongly correlated to the forward speed of the robot. The more tail height the larger speed value. On the other hand, the influence of the cargo is inversely proportional to the forward speed. The proposed prototype demonstrates to carry large amounts of cargo. However, the forward speed reduces drastically. Besides, the boundary effect was investigated using three multiflagella prototypes with a symmetric tail distribution. For that experiment, two containers with 20 and $50 \mathrm{~mm}$ in width were used. Simulation and experiments demonstrates an increment on propulsion speed at same frequencies when robots are immersed in the container of smaller width. Experimentally we achieved a relative increment up to $59 \%$. Nonetheless, the cut-off frequencies is reduced since the fluidic torque increases in the small container. Finally, the effectiveness of the multi-tailed robots was discussed showing that the 2tailed robot was the best one from our experiments. The next step of this work is to scale down our flagella prototypes in order to see their viability for microscale applications.

\section{REFERENCES}

[1] Howard C Berg and Linda Turner. Chemotaxis of bacteria in glass capillary arrays. escherichia coli, motility, microchannel plate, and light scattering. Biophysical Journal, 58(4):919-930, 1990.

[2] Bram Bet, Gijs Boosten, Marjolein Dijkstra, and René van Roij. Efficient shapes for microswimming: From three-body swimmers to helical flagella. The Journal of chemical physics, 146(8):084904, 2017.
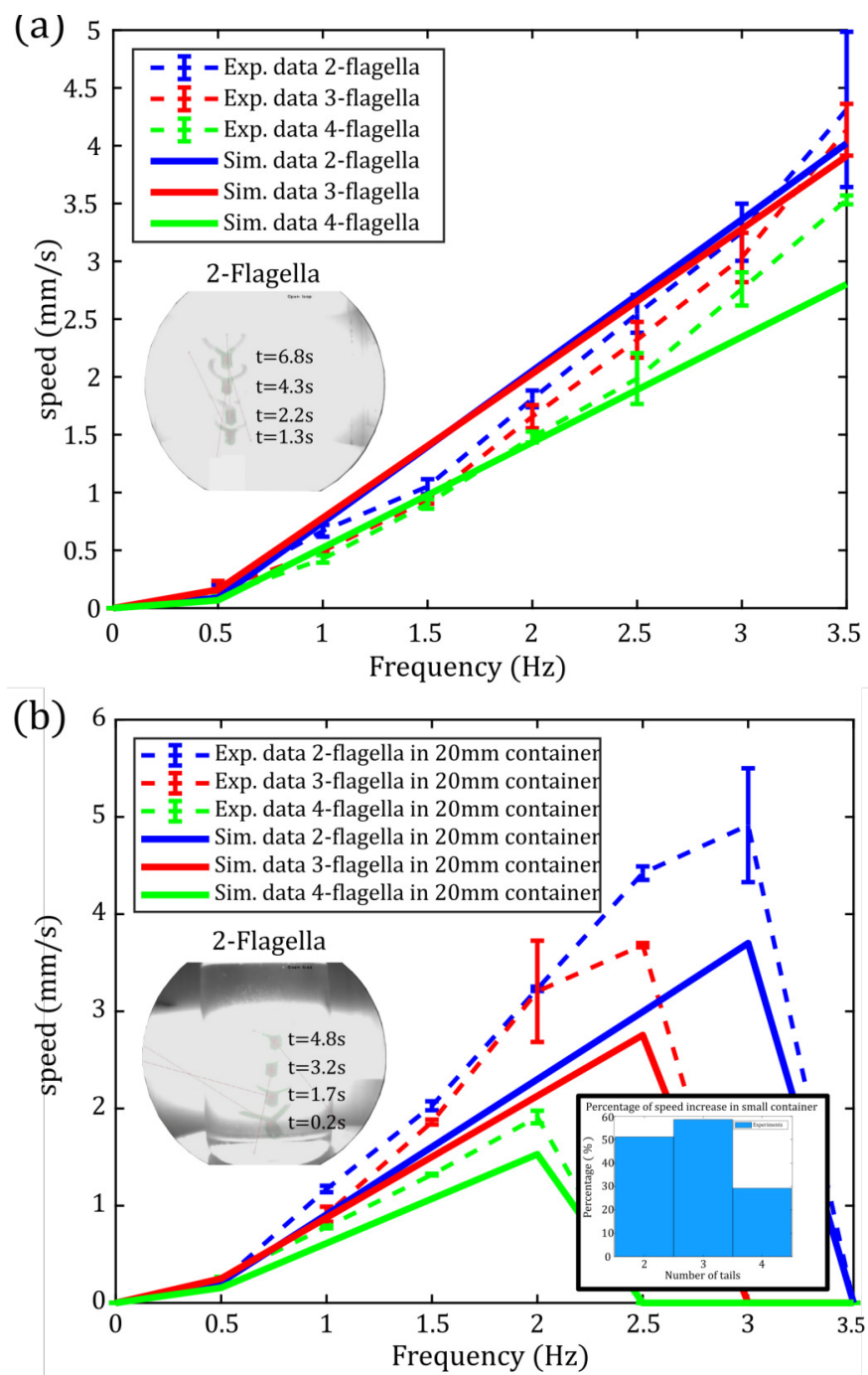

Fig. 9. Confinement effect, (a) Robots with 2 (blue), 3 (red) and 4 (green) tails are assessed in a 50-mm in width container. The 2 and 3-tailed robots have close speed values. (b) The robots are tested in a $20-\mathrm{mm}$ in width container experiencing cut-off frequencies because of the increase of the fluid torque associated to the degree of confinement. The inset on the bottom left show the percentage of speed increment at cut-off frequencies of each multi-flagellated robot. Being the 3-tailed robot the one who experiences a percentage up to 59\%.The experimental error bars are calculated as the standard deviation of three trials for each frequency and robot.

[3] Sherri A Biondi, John A Quinn, and Howard Goldfine. Random motility of swimming bacteria in restricted geometries. AIChE Journal, 44(8):1923-1929, 1998.

[4] Hakan O Caldag, Alperen Acemoglu, and Serhat Yesilyurt. Experimental characterization of helical swimming trajectories in circular channels. Microfluidics and Nanofluidics, 21(8):136, 2017.

[5] Rika Wright Carlsen, Matthew R Edwards, Jiang Zhuang, Cecile Pacoret, and Metin Sitti. Magnetic steering control of multi-cellular bio-hybrid microswimmers. Lab on a Chip, 14(19):3850-3859, 2014.

[6] Umit Danis, Reza Rasooli, Chia-Yuan Chen, Onur Dur, Metin Sitti, and Kerem Pekkan. Thrust and hydrodynamic efficiency of the bundled flagella. Micromachines, 10(7):449, 2019.

[7] Henry C Fu, Mehdi Jabbarzadeh, and Farshad Meshkati. Magnetization directions and geometries of helical microswimmers for linear velocity-frequency response. Physical Review E, 91(4):043011, 2015.

[8] Tatsuya Fujita and Tatsuo Kawai. Optimum shape of a flagellated microorganism. JSME International Journal Series C Mechanical Systems, Machine Elements and Manufacturing, 44(4):952-957, 2001.

[9] Eric Lauga and Thomas R Powers. The hydrodynamics of swimming 
microorganisms. Reports on Progress in Physics, 72(9):096601, 2009.

[10] Christopher $\mathrm{T}$ Lefèvre, Alain Bernadac, Kui Yu-Zhang, Nathalie Pradel, and Long-Fei Wu. Isolation and characterization of a magnetotactic bacterial culture from the mediterranean sea. Environmental microbiology, 11(7):1646-1657, 2009.

[11] Bin Liu, Kenneth S Breuer, and Thomas R Powers. Propulsion by a helical flagellum in a capillary tube. Physics of Fluids, 26(1):011701, 2014.

[12] Edward M. Purcell. Life at low Reynolds number. American Journal of Physics, 45(1):3-11, January 1977.

[13] Juanfang Ruan, Takayuki Kato, Claire-Lise Santini, Tomoko Miyata, Akihiro Kawamoto, Wei-Jia Zhang, Alain Bernadac, Long-Fei Wu, and Keiichi Namba. Architecture of a flagellar apparatus in the fastswimming magnetotactic bacterium mo-1. Proceedings of the National Academy of Sciences, 109(50):20643-20648, 2012.

[14] Henry Shum. Microswimmer propulsion by two steadily rotating helical flagella. Micromachines, 10(1):65, 2019.

[15] John Singleton, Eric Diller, Tim Andersen, Stéphane Regnier, and Metin Sitti. Micro-scale propulsion using multiple flexible artificial flagella. In 2011 IEEE/RSJ International Conference on Intelligent Robots and Systems, pages 1687-1692. IEEE, 2011.

[16] Hirofumi Wada and Roland R Netz. Model for self-propulsive helical filaments: kink-pair propagation. Physical review letters, 99(10):108102, 2007.

[17] Tiantian Xu, Gilgueng Hwang, Nicolas Andreff, and Stéphane Régnier. Influence of geometry on swimming performance of helical swimmers using doe. Journal of Micro-Bio Robotics, 11(1-4):57-66, 2016.

[18] Jing Yang, Charles W Wolgemuth, Greg Huber, et al. Kinematics of the swimming of spiroplasma. Physical review letters, 102(21):218102, 2009.

[19] Zhou Ye, Stéphane Régnier, and Metin Sitti. Rotating magnetic miniature swimming robots with multiple flexible flagella. IEEE Transactions on Robotics, 30(1):3-13, 2013. 